\title{
Salivary IgA and periodontal treatment needs in diabetic patients
}

\section{Luciana Salles Branco-de- Almeida $^{(a)}$ Cláudia Maria Coêlho Alves ${ }^{(b)}$ Fernanda Ferreira Lopes ${ }^{(b)}$ Adriana de Fátima Vasconcelos Pereira(b) Rosane Nassar Meireles Guerra(c) Antônio Luiz Amaral Pereira ${ }^{(b)}$}

(a) Postgraduate Department, University Center of Maranhão, São Luís, MA, Brazil.

(b) Department of Dentistry II, Federal University of Maranhão, São Luís, MA, Brazil.

(c)Department of Pathology, Federal University of Maranhão, São Luís, MA, Brazil.
Declaration of Interests: The authors certify that they have no commercial or associative interest that represents a conflict of interest in connection with the manuscript.

Corresponding author:

Antônio Luiz Amaral Pereira

E-mail:alap@ufma.br

Received for publication on Jun 05, 2011 Accepted for publication on Sep 14, 2011
Abstract: Salivary IgA can serve as a first line of defense against pathogens that colonize and invade mucosal surfaces and may be protective against periodontal disease. The aim of this study was to assess salivary immunoglobulin A levels in diabetic and non-diabetic patients with different periodontal treatment needs. The Periodontal Screening \& Recording (PSR) system was used to evaluate the periodontal treatment needs of 41 diabetic and 42 non-diabetic patients. Unstimulated whole saliva was collected from each patient immediately before clinical examination and stored at $-20^{\circ} \mathrm{C}$ until analysis. Salivary immunoglobulin A (s-IgA) levels were determined using an enzyme-linked immunosorbent assay, and values were expressed as optical density. Diabetic and non-diabetic patients were compared using clinical and laboratory data. PSR data indicated that periodontal disease was more frequent and more severe in diabetic patients. A higher prevalence of codes 3 and 4 was observed in diabetics as compared with non-diabetics (odds ratio $=2, P<0.05$ ). Furthermore, non-diabetic patients had more healthy sextants (code 0$)$ than did diabetic patients. Over half of diabetic patients $(\sim 54 \%)$ presented with s-IgA levels that were lower than the normal range (optical density from $0.4 \mathrm{~nm}$ to $0.6 \mathrm{~nm} ; P<0.05)$. In addition, diabetic patients showed a higher variability in s-IgA levels as compared with non-diabetic patients. In conclusion, diabetic individuals had lower s-IgA levels, more-frequent and more-severe periodontal disease, and a greater need for periodontal treatment as compared with non-diabetic patients.

Descriptors: Periodontal Diseases; Diabetes Mellitus; Immunoglobulin A.

\section{Introduction}

Periodontal disease (PD) is a multi-factorial disease that encompasses hard and soft dental supporting tissues, microbial colonization, and host immune/inflammatory responses. The humoral host immune responses play an important role in protection of the oral environment because of the ability of antibodies to inhibit microbial attachment to cell surfaces and aggregation/opsonization of microorganisms. ${ }^{1}$ In addition, antibodies are associated with complementation of alternative pathways that are also important in preventing colonization and promoting lysis of microorganisms, as well as neutralizing toxic products in the context of PD., ${ }^{1,2}$

Specific antibody systems, including those that include immunoglobu$\operatorname{lin} \mathrm{A}(\operatorname{Ig} \mathrm{A})$, are found in saliva, which is a complex body fluid that is in- 
creasingly used for early diagnosis and detection of potential vulnerability to several diseases. ${ }^{3,4}$ In this context, analysis of whole saliva provides a simple and non-invasive method of evaluating the role of salivary IgA (s-IgA) levels in PD. ${ }^{5-7}$ s-IgA represents the first line of defense against pathogens that colonize and invade mucosal surfaces, thus interfering with the development of PD at a very early, but very critical, stage. ${ }^{8}$ Studies have suggested a protective role for s-IgA in patients with PD, ${ }^{5,9,10}$ and low concentrations of s-IgA have been associated with severe forms of this disease. ${ }^{11}$

Systemic diseases such as diabetes mellitus (DM) can modulate the host immune reactions against PD. The relationship between DM and PD has been widely reported. ${ }^{12,13} \mathrm{DM}$ is a complex disease comprising significant metabolic disorders and defective host immune responses, which influence the development and severity of PD. ${ }^{13}$ The role of s-IgA levels in the susceptibility to PD among diabetic patients is not, however, well understood, and existing studies have shown contradictory results. ${ }^{14-16}$

Based on the importance of s- $\operatorname{Ig} \mathrm{A}$ as a host defense factor as well as the incomplete understanding concerning the role of $\mathrm{s}-\operatorname{IgA}$ in the pathogenesis of PD in patients with DM, the aim of this study was to assess both the periodontal treatment needs and the s-IgA levels in diabetic and non-diabetic individuals.

\section{Methodology \\ Study group and patient selection}

Diabetic patients were recruited from the Endocrinology Section of the Federal University of Maranhão Hospital (São Luís, Brazil). Non-diabetic patients (control group) were selected from patients from the same hospital with no chronic or autoimmune diseases. All patients were in agreement with the protocol approved by the Brazilian ethics committee (Protocol No 00354/2003).

Initially, patients answered a social demographic questionnaire, which allowed for identification and further exclusion of individuals with other systemic diseases. Written informed consent was obtained from all participants in the study.

\section{Collection of saliva samples}

All saliva samples were taken prior to clinical examination at 9:00 a.m. and 12:00 p.m. by a single trained examiner. Unstimulated whole saliva samples were collected by spitting into an icecooled receptacle. ${ }^{17}$ Saliva samples were centrifuged (11,900 rpm, $10 \mathrm{~min})$, and supernatants were stored at $-20^{\circ} \mathrm{C}$ until analysis.

\section{Clinical examination}

The periodontal status of the patients was assessed using the Periodontal Screening and Recording (PSR) system, which is a diagnostic screening tool recommended by the American Academy of Periodontology (AAP) for the early detection of PD. ${ }^{18}$ A single trained examiner performed the clinical evaluation using a WHO-621 probe, ${ }^{18}$ which contains a colored band $(3.5-5.5 \mathrm{~mm})$ and a 0.5 $\mathrm{mm}$ sphere on its active end. The mouth of each patient was divided into six sextants (S1-S6), beginning in the maxillary right sextant (S1), proceeding in a clockwise direction, and finishing in the mandibular right sextant (S6). A code $(0,1,2,3$, or 4$)$ was assigned to each sextant based on the highest probing value obtained from any tooth in that sextant. ${ }^{18}$ All codes and the highest PSR code for each sextant were recorded (edentulous sextants were recorded as "X"). Following data collection, patients received oral hygiene instructions and information on their periodontal condition and treatment needs according to their PSR codes. ${ }^{18}$

\section{Determination of $s-\lg A$ levels}

Total s-IgA levels were determined using an enzyme-linked immunosorbent assay (ELISA) as described. ${ }^{19}$ Briefly, anti-human IgA (Sigma, Saint Louis, USA) was diluted to a concentration of $50 \mu \mathrm{g} / \mathrm{mL}$ in carbonate-bicarbonate buffer ( $\mathrm{pH}$ 9.6) and adsorbed onto wells of flat-bottomed ELISA strips (NUNC, Roskilde, Denmark) with overnight incubation at $4{ }^{\circ} \mathrm{C}$. The wells were then washed five times with $0.15 \mathrm{M}$ phosphate-buffered saline (PBS; $\mathrm{pH}$ 7.2) containing $0.05 \%$ Tween 20 (Promega, Madison, USA; PBS-T) and incubated in PBS containing $1 \%$ bovine serum albumin (Sigma; PBS-TA) for $1 \mathrm{~h}$ at $37^{\circ} \mathrm{C}$. Wells were washed five times, fol- 
lowed by the addition of $100 \mu \mathrm{L}$ of 10 -fold dilutions (1:100 to $1: 1000)$ of saliva samples in PBS. After $30 \mathrm{~min}$ at $37^{\circ} \mathrm{C}$ and further washing, $100 \mu \mathrm{L}$ alkaline phosphatase-labeled goat anti-human IgA (Sigma; diluted 1:1000 in PBS-T) was added to the wells and incubated for $2 \mathrm{~h}$ at $37^{\circ} \mathrm{C}$. The strips were then washed five times with PBS-T, and the color was developed by adding $100 \mu \mathrm{L}$ of a solution of $\rho$-nitrophenylphosphate (Sigma; pNPP) to the strips and incubating them in the dark for $30 \mathrm{~min}$. The reaction was stopped with $1 \mathrm{~N} \mathrm{NaOH}$ (Sigma), and the optical density was measured at a wavelength of $405 \mathrm{~nm}$.

\section{Statistical analysis}

Differences in the frequencies of PSR codes were estimated by the Fisher's exact test. The odds ratio (OR) and the $95 \%$ confidence intervals (CI) associated with the different groups were also calculated. Normality of distribution of s-IgA levels (expressed as optical density values) was checked using the Kolmogorov-Smirnov test. Statistical differences between the measurements of s-IgA from diabetics and non-diabetics were analyzed using the Student's $t$-test. Significance was considered at $P<0.05$. Statistical analyses were performed using BioEstat 5.0 (Sociedade Civil Mamirauá, Belém, Brazil).

\section{Results}

\section{Clinical evaluation}

We examined 41 diabetic individuals (12 with type $1 \mathrm{DM}$ and 29 with type $2 \mathrm{DM}$; mean age, $57.1 \pm 13.9$ years; 29 women and 12 men) and 42 non-diabetic individuals (mean age, $30.0 \pm 10.3$ years; 37 women and 4 men). PSR codes and distribution of sextants with each code in diabetic and non-diabetic individuals are shown in Table 1. In the diabetic group, all patients exhibited some degree of periodontal pathological changes (data not shown). A higher prevalence of sextants with severe periodontitis (code 4) was observed in diabetic patients as compared with non-diabetic patients (Table 1). In addition, the presence of fewer healthy sextants was detected in diabetics (Table 1). Comparison between the frequencies of codes found in diabetics and nondiabetics indicated a more severe periodontal condition in diabetic patients (greater prevalence of codes 3 and 4) as compared with non-diabetics ( $\mathrm{OR}=2$, $P<0.05$; Table 2). Altogether, PSR data demonstrated a higher severity of PD in diabetics as compared with non-diabetics.

\section{Total IgA levels in saliva}

s-IgA levels of non-diabetic (control group) and diabetic individuals are shown in Figure 1 (A and B, respectively). Greater variability in s-IgA levels was observed in the diabetic group $(0.148-1.172 \mathrm{~nm})$ as compared with levels in the non-diabetic group (0.321 - $0.795 \mathrm{~nm}$; Figure 1). Based on the normal range for $\mathrm{s}-\mathrm{IgA}$, the following distribution was observed for diabetic individuals: $53.6 \%$ (22 individuals) showed low s-IgA levels $(<0.4 \mathrm{~nm}), 36.6 \%$ of

Table 1 - PSR codes and total distribution of the number of sextants (n) with each code in diabetic and non-diabetic patients.

\begin{tabular}{c|c|r|r|r}
\hline \multirow{2}{*}{ PSR codes } & \multicolumn{2}{|c|}{ Diabetics } & \multicolumn{2}{c}{ Non-diabetics } \\
\cline { 2 - 5 } & $n$ & $f(\%)^{a}$ & \multicolumn{1}{c}{$n$} & $f(\%)^{\circ}$ \\
\hline 0 & 17 & 6.9 & 33 & 13.1 \\
\hline 1 & 27 & 11.0 & 97 & 38.5 \\
\hline 2 & 77 & 31.3 & 77 & 30.6 \\
\hline 3 & 29 & 11.8 & 28 & 11.1 \\
\hline 4 & 10 & 4.1 & 5 & 2.0 \\
\hline$X$ & 86 & 35.0 & 12 & 4.8 \\
\hline
\end{tabular}

of (\%), frequency expressed as percentage.

Table 2 - Comparison of PSR codes in diabetics and nondiabetics.

\begin{tabular}{|c|c|c|c|c|c|c|c|c|}
\hline \multirow{3}{*}{ Group } & \multicolumn{6}{|c|}{ PSR codes } & \multirow{3}{*}{$P$ value } & \multirow{3}{*}{$\begin{array}{l}\text { OR and } \\
95 \% \mathrm{Cl}^{c}\end{array}$} \\
\hline & \multicolumn{2}{|c|}{0 to 2} & \multicolumn{2}{|c|}{3 to 4} & \multicolumn{2}{|c|}{ Total } & & \\
\hline & $n$ & $f(\%)^{a}$ & $\mathrm{n}$ & $f(\%)^{a}$ & $n$ & $f(\%)^{a}$ & & \\
\hline Diabetics & 121 & 75.6 & 39 & 24.4 & 160 & 100 & \multirow{2}{*}{$0.0079^{b}$} & \multirow{2}{*}{$\begin{array}{c}2.02 \\
(1.21-3.38)\end{array}$} \\
\hline Non-diabetics & 207 & 86.2 & 33 & 13.8 & 240 & 100 & & \\
\hline
\end{tabular}

a $\mathrm{f}(\%)$, frequency expressed as percentage; ${ }^{\mathrm{b}}$ Fisher's exact test; ${ }^{\mathrm{c}} \mathrm{OR}$, odds ratio; $\mathrm{Cl}$, confidence interval. 


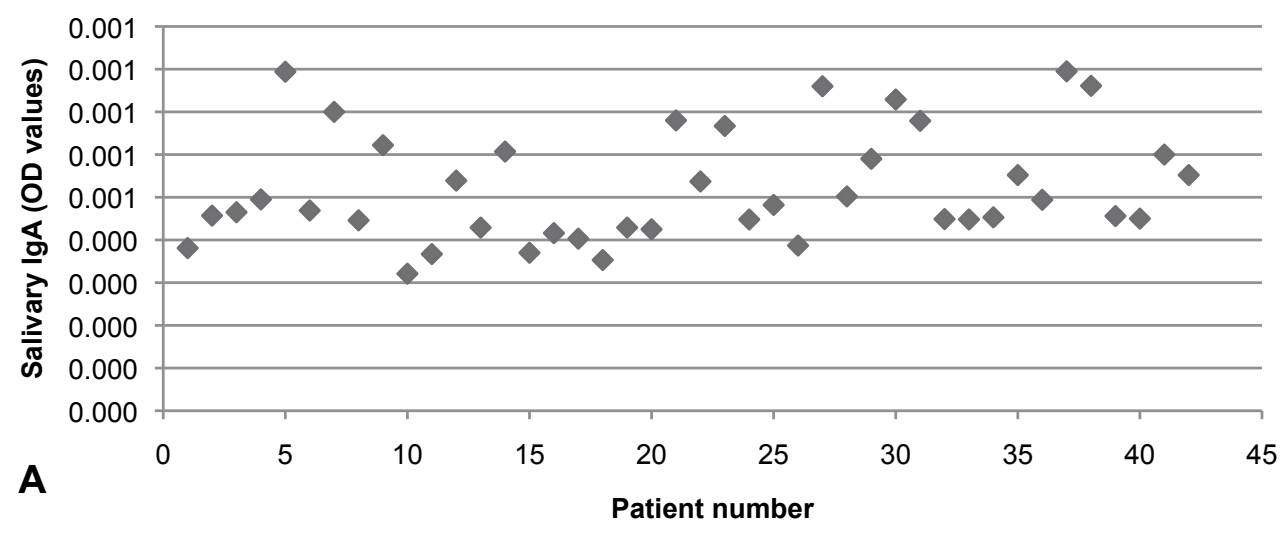

Non-diabetics (control)

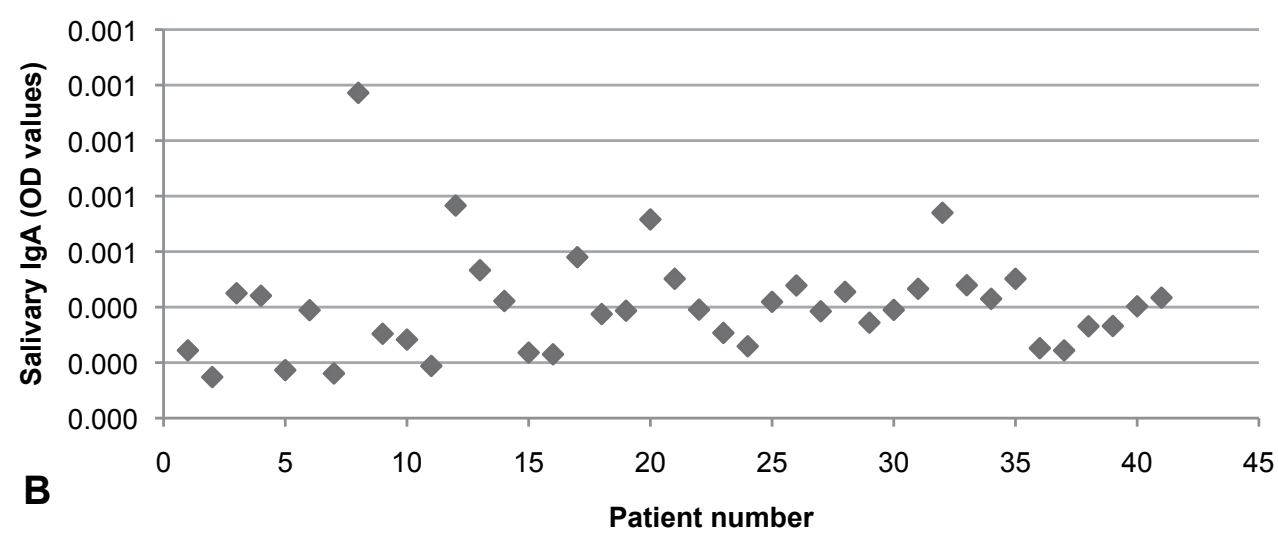

Diabetics

Figure 1 - Salivary IgA levels (OD values) of non-diabetic (A) and diabetic (B) patients as determined by ELISA.

patients (15 individuals) had s-IgA levels within the normal range, and 9.8\% (4 individuals) presented with high s-IgA levels $(>0.6 \mathrm{~nm} ; P<0.05$ for all comparisons with control individuals; Figure 2). Thus, in our current study, diabetic patients expressed lower levels of s-IgA as compared with nondiabetic individuals.

\section{Discussion}

This investigation showed that diabetic individuals had more-severe PD and lower s-IgA levels as compared with non-diabetic individuals. These findings are important because (1) previous studies have not determined whether s-IgA has a protective or detrimental effect on PD prevalence and severity, and (2) there are few human studies on the role of s-IgA with respect to the susceptibility to PD among diabetic individuals. Our data suggest that s-IgA may be protective against $\mathrm{PD}$, because its levels were reduced in patients with more-severe PD (i.e., diabetics).

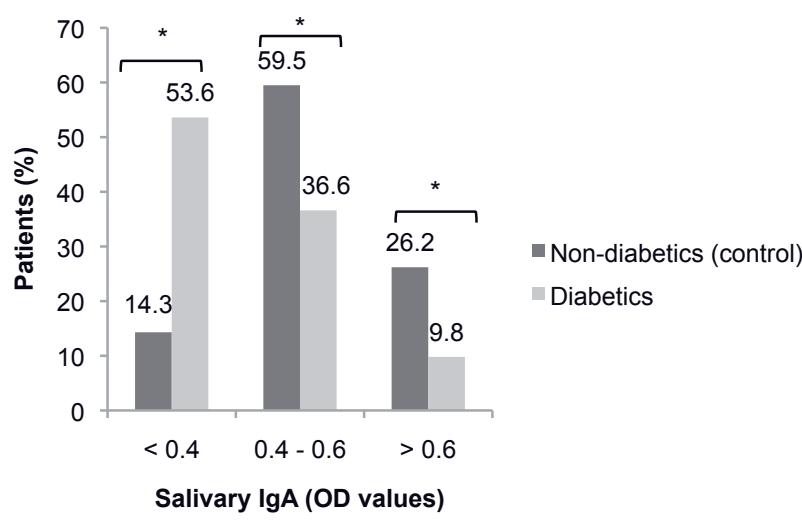

Figure 2 - Distribution of non-diabetic (control group) and diabetic patients according to salivary $\lg A$ levels as determined by ELISA (*, $P<0.05$; Student's $t$-test). Numbers above each bar indicate the actual percentage.

We used the PSR system ${ }^{18}$ to investigate the periodontal treatment needs of patients. PSR, a simplified probing technique, was designed to facilitate and expedite screening for PD. PSR is considered a quick, reliable, and reproducible method for iden- 
tifying the treatment needs of patients. ${ }^{20}$ PSR is a valuable screening tool for PD, because it generates measurements that are significantly correlated with the probing depths and attachment level measurements obtained with traditional clinical periodontal examinations. ${ }^{21,22}$

The higher severity of PD among diabetic individuals that was observed in our current study was detected by the presence of a greater number of oral sextants with periodontitis (codes 3 and 4), fewer healthy sextants, and more tooth loss (edentulous sextants) as compared with non-diabetics (control group) as determined by the PSR system. The relationship between DM and PD has been widely reported, and our current findings are in accordance with previous investigations that have evaluated the prevalence or severity of PD in diabetics. ${ }^{12,23,24}$ Although non-diabetic patients were periodontally healthier than were diabetics, only one patient in our study, a non-diabetic individual, was devoid of periodontal pathological changes.

We also evaluated the relationship between PD and the total s-IgA values in diabetic and non-diabetic patients. s-IgA is the predominant immunoglobulin secreted in oral mucosal sites and has been considered the major factor that contributes to mucosal health and microbial defense. ${ }^{25}$ The total IgA content in saliva is a mixture of (1) dimeric secretory IgA derived from plasma cells present in the salivary glands and (2) monomeric IgA, which originates from plasma cells present in the gingival crevicular fluid. ${ }^{25,26}$ Thus, analysis of whole saliva holds greater promise than gland-specific saliva as a diagnostic tool for PD. ${ }^{27}$ Determination of possible alterations in whole saliva composition may be helpful for understanding the increased severity of PD in diabetic patients, as well as for diagnosing possible variations and defective host immune responses, including the secretion of s-IgA. ${ }^{6,28}$

The predominance of low s-IgA values in diabetics that we observed may be related to the fact that to be effective, antibodies require the cooperation of other host-protective mechanisms. In DM patients, other immune components, such as polymorphonuclear leukocytes, are not fully functional. ${ }^{13}$ In contrast, a greater variability in s-IgA levels was found in diabetics as compared with non-diabetics, which is probably related to the complex host immune responses to $\mathrm{PD}$ that are observed in diabetic individuals. ${ }^{13}$ In addition, considering the many wellknown factors that are implicated in the development of $\mathrm{PD}$, such as variations in s-IgA, our results are not surprising. These multiple factors may also explain the absence of a correlation between clinical and laboratory data observed in our current study (data not shown).

Our findings support the idea that s-IgA may play a protective role in the periodontium, because s-IgA levels were lower in the diabetic group, which had more severe periodontal changes. Other studies have shown similar results. For example, Lamster et al. ${ }^{29}$ showed that patients with clinical attachment loss have lower concentrations of $\operatorname{IgA}$ in their gingival crevicular fluid. Plombas et al. ${ }^{30}$ found that individuals with periodontitis have lower levels of specific s-IgA than do healthy patients. In addition, Hägewald et al. ${ }^{11}$ observed that levels of total $\operatorname{IgA}$ are reduced in patients with aggressive periodontitis. Thus, our findings and previous studies suggest that the levels of s-IgA may positively influence the clinical parameters and severity of PD.

\section{Conclusions}

In conclusion, our current study shows a greater need for periodontal treatment in diabetics. Lower s-IgA levels among diabetics as compared with those among non-diabetics indicated that PD was more prevalent and more severe in patients with low levels of s-IgA. In addition, diabetic individuals had more-variable s-IgA values, which may be related to the influence of the complex host immune responses on both PD and DM.

\section{Acknowledgements}

The authors are grateful to the members of the Diabetes Assistance Program (Endocrinology Section, Hospital of Federal University of Maranhão, Brazil) for technical support. The authors thank the Brazilian Government Agency for a scholarship to the first author (CNPq 104829/00-1). 


\section{References}

1. Teng YT. Protective and destructive immunity in the periodontium: Part 1--innate and humoral immunity and the periodontium. J Dent Res. 2006 Mar;85(3):198-208.

2. Albandar JM, DeNardin AM, Adesanya MR, Diehl SR, Winn DM. Associations between serum antibody levels to periodontal pathogens and early-onset periodontitis. J Periodontol. 2001 Nov;72(11):1463-9.

3. Streckfus CF, Bigler LR. Saliva as a diagnostic fluid. Oral Dis. 2002 Mar;8(2):69-76.

4. Lee YH, Wong DT. Saliva: an emerging biofluid for early detection of diseases. Am J Dent. 2009 Aug;22(4):241-8.

5. Grbic JT, Lamster IB, Fine JB, Lam KS, Celenti RS, HerreraAbreu M, et al.. Changes in gingival crevicular fluid levels of immunoglobulin A following therapy: association with attachment loss. J Periodontol. 1999 Oct;70(10):1221-7.

6. Bachrach G, Muster Z, Raz I, Chaushu G, Stabholz A, Nussbaum $\mathrm{G}$, et al.. Assessing the levels of immunoglobulins in the saliva of diabetic individuals with periodontitis using checkerboard immunodetection. Oral Dis. 2008 Jan;14(1):51-9.

7. Giannobile WV, Beikler T, Kinney JS, Ramseier CA, Morelli T, Wong DT. Saliva as a diagnostic tool for periodontal disease: current state and future directions. Periodontol 2000. 2009;50(1):52-64.

8. McNabb PC, Tomasi TB. Host defense mechanisms at mucosal surfaces. Annu Rev Microbiol. 1981;35:477-96.

9. Schenck K, Poppelsdorf D, Denis C, Tollefsen T. Levels of salivary IgA antibodies reactive with bacteria from dental plaque are associated with susceptibility to experimental gingivitis. J Clin Periodontol. 1993 Jul;20(6):411-7.

10. Grbic JT, Singer RE, Jans HH, Celenti RS, Lamster IB. Immunoglobulin isotypes in gingival crevicular fluid: possible protective role of IgA. J Periodontol. 1995 Jan;66(1):55-61.

11. Hägewald S, Bernimoulin JP, Köttgen E, Kage A. Salivary IgA subclasses and bacteria-reactive IgA in patients with aggressive periodontitis. J Periodontal Res. 2002 Oct;37(5):333-9.

12. Soskolne WA, Klinger A. The relationship between periodontal diseases and diabetes: an overview. Ann Periodontol. 2001 Dec;6(1):91-8.

13. Ryan ME, Carnu O, Kamer A. The influence of diabetes on the periodontal tissues. J Am Dent Assoc. 2003 Oct;134 Spec No:34S-40S.

14. Tenovuo J, Lehtonen OP, Viikari J, Larjava H, Vilja P, Tuohimaa P. Immunoglobulins and innate antimicrobial factors in whole saliva of patients with insulin-dependent diabetes mellitus. J Dent Res. 1986 Jan;65(1):62-6.

15. Ben-Aryeh H, Cohen M, Kanter Y, Szargel R, Laufer D. Salivary composition in diabetic patients. J Diabet Complications. 1988 Apr-Jun;2(2):96-9.

16. Streckfus CF, Marcus S, Welsh S, Brown RS, Cherry-Peppers G, Brown RH. Parotid function and composition of parotid saliva among elderly edentulous African-American diabetics. J Oral Pathol Med. 1994 Jul;23(6):277-9.

17. Henskens YM, van den Keijbus PA, Veerman EC, Van der Weijden GA, Timmerman MF, Snoek CM, et al. Protein composition of whole and parotid saliva in healthy and periodontitis subjects. Determination of cystatins, albumin, amylase and IgA. J Periodontal Res. 1996 Jan;31(1):57-65.

18. Periodontal screening and recording training program kit. Chicago: American Dental Association and American Academy of Periodontics; 1992.

19. Guerra RN, Silva VM, Aragão-França LS, Oliveira PR, Feitosa R, Nascimento FR, et al.. Babassu aqueous extract (BAE) as an adjuvant for $\mathrm{T}$ helper (Th)1-dependent immune responses in mice of a Th2 immune response-prone strain. BMC Immunol. 2011 Jan 29;12:13.

20. Covington LL, Breault LG, Hokett SD. The application of Periodontal Screening and Recording (PSR) in a military population. J Contemp Dent Pract. 2003 Aug 15;4(3):36-51.

21. Khocht A, Zohn H, Deasy M, Chang KM. Assessment of periodontal status with PSR and traditional clinical periodontal examination. J Am Dent Assoc. 1995 Dec;126(12):1658-65.

22. Khocht A, Zohn H, Deasy M, Chang KM. Screening for periodontal disease: radiographs vs. PSR. J Am Dent Assoc. 1996 Jun;127(6):749-56.

23. Emrich LJ, Shlossman M, Genco RJ. Periodontal disease in non-insulin-dependent diabetes mellitus. J Periodontol. 1991 Feb;62(2):161-3.

24. Firatli E. The relationship between clinical periodontal status and insulin-dependent diabetes mellitus. Results after 5 years. J Periodontol. 1997 Feb;68(2):136-40.

25. Genco RJ. Host responses in periodontal diseases: current concepts. J Periodontol. 1992 Apr;63(4 Suppl):338-55.

26. Hägewald S, Bernimoulin JP, Köttgen E, Kage A. Total IgA and Porphyromonas gingivalis-reactive IgA in the saliva of patients with generalized early-onset periodontitis. Eur J Oral Sci. 2000 Apr;108(2):147-53.

27. Kaufman E, Lamster IB. Analysis of saliva for periodontal diagnosis--a review. J Clin Periodontol. 2000 Jul;27(7):45365.

28. Belazi MA, Galli-Tsinopoulou A, Drakoulakos D, Fleva A, Papanayiotou PH. Salivary alterations in insulin-dependent diabetes mellitus. Int J Paediatr Dent. 1998 Mar;8(1):29-33.

29. Lamster IB, Smith QT, Celenti RS, Singer RE, Grbic JT. Development of a risk profile for periodontal disease: microbial and host response factors. J Periodontol. 1994 May;65(5 Suppl):511-20.

30. Plombas M, Gobert B, De March AK, Sarda MN, Sixou $\mathrm{M}$, Béné $\mathrm{MC}$, et al.. Isotypic antibody response to plaque anaerobes in periodontal disease. J Periodontol. 2002 Dec;73(12):1507-11. 